\title{
A Review on Black hole solutions in General Relativity
}

\author{
Monika Sati ${ }^{1}$ • K.C. Petwal ${ }^{2}$ \\ ${ }^{1}$ Department of Mathematics, H.N.B. Garhwal University (A Central University), S.R.T Campus Badshahithaul, \\ Tehri Garhwal, Uttarakhand, India \\ *Correspondence author: monikasati123@gmail.com
}

Received: 28.6.2021; Revised: 10.11.2021; Accepted:20.11.2021

\begin{abstract}
In the present manuscript, we endeavour to review and developthe black hole solutions in general relativity. We emphasizehere the Schwarzschild solution in Einstein's field equation, which describes the gravitational field outside a spherical mass. The paper aims to obtain certain results, including the description of the Einsteinfield equationwith stationary and static solutions and components of the metric that turns out to be timeindependent, some experiments on the Schwarzschild - Penrose diagram, theKerr-Newman solution for rotating black holes, and the Reissner- Nordstrom solution for static and charged black holes.
\end{abstract}

Keywords: General Relativity, Einstein equation, Schwarzschild metric, Kerr-Newman, Reissner-Nordstrom

MSC 2010: 83Cxx, 83C05, 83C22, 83C57

\section{Introduction}

Einstein's gravitational principle is expressed in a deceptively simple-looking tensor equation (scalar and vector generalization) that describes how a mass defines the curvature of spacetime around it. One of the most interesting findings is the solution to the equation: the black hole. The hypothesis states that if an object is dense enough, it will collapse in on itself and be surrounded by a continuum of events from which nothing can escape [5]. The term "black hole", invented by astronomer John Wheeler in 1969 , refers to the fact that light cannot escape such an object. Karl Schwarzschild was the first to mention this phenomenon in 1916 , but at that time it was mainly considered a novelty in mathematics.

According to Newton's theory of gravity, the escape velocity $v$ from a distance $r$ for the center of gravity of a heavy object of mass $M$ is:

$$
\frac{1}{2} v^{2}=\frac{G M}{r}
$$

Where $G$ is the gravitational constant. What happens if a body with a large mass $M$ is so compact that the escape velocity from its surface exceeds that of light, or, $v>c$ ? There are bodies of mass $M$ and radius $R$ for which holds [1].

$$
\frac{2 G M r}{c^{2}} \geq 1
$$

John Mitchell had already raised this question in 1783. In 1796, the case was further investigated by Pierri Simon de Laplace. Do light rays fall back on the surface of an object in this way? Only light cannot disappear in space, one might think. Later it was conjectured that it could to escape anyway because of the wave nature of light. 
Now we know that such simple assumptions are fallacious. To understand what happens to these extremely heavy objects, one must consider Einstein's theory of relativity [1], both Special Relativity and General Relativity, the theory that defines the gravitational field when velocities are generated compared to those of light. Soon after the formulation of this beautiful theory by Albert Einstein, it was realized that his equations have closed solutions. One naturally first tries to find solutions with maximum symmetry, which the radially symmetric case. For later more general solutions with less symmetry were also found. However, these solutions had some features that were difficult to understand at first. Singularities seemed impossible to recognize as physical phenomena until it was discovered that at least some of these singularities were due only to externalities. Upon closer examination, it turned out what their true physical nature was. It turned out that a space traveller could go all the way in such a "thing" but would never return, at least in principle. In fact, even the light from these solutions would not exit the central area. It was John Archibold Wheeler who called these strange things "black holes". Einstein was not satisfied with this. Like others at first, he assumed that these unusual properties were due to poor, or at least incomplete, physical perception. Those crazy black holes will go down all right, he thought to himself. But his equations are much better understood today. Not only do we accept the existence of black holes, but we also understand how they can form under different circumstances. The theory helps quantify the behaviour of material objects, surfaces, or other factors near or beyond a black hole. Moreover, astronomers have now found several bodies in the universe that are completely consistent with the theorists' thorough explanations. These objects can only be interpreted as black holes, like everything else [5].

The "astronomical black holes" display no clash with other physical laws at all. Indeed, under intense circumstances, they've become a rich source of information about physical phenomena. General Relativity itself can now also be examined to great precision. Astronomers find that black holes would only form from regular stellar bodies. If they contain a minimum mass, becoming process is known for low mass black holes, and indeed no indications have been found that black holes exist anywhere in the universe much lighter than this "Chandrasekhar limit"[2].

One of the most important findings of the theory of General Relativity is the presence of black holes. Also if certain elements of black hole physics can be drawn from Newtonian theory, the entire idea of black holes requires the application of an event horizon and a traditional relativistic principle causal mechanism.

Maybe we are close to a definite discovery of black holes, and there is an abundance of data on black holes. Within General Relativity, there are four most well-known black hole solutions: the uncharged static black hole provided by the Schwarchild solution, the charged static black hole; the ReissnerNordstrom solution; the uncharged black hole, the Kerr solution and the revolving black hole, the Kerr- Newman solution [9]. 
The characteristics of these solutions led to the so-called no- hair conjectures: in general, only three parameters in Einstein-Maxwell theory mass $M$, charge $Q$ and angular momentum $I$ must be characterized by black hole; however, additional parameters relating to new charges are applied as more gauge fields are taken into account as happens, for example, for Lagrangian induced by string theory, other parameters such as magnetic monopole charges can also occur when taking into account other general gauge fields. There is clear proof that in some cases this hypothesis can be broken, in particular when considering new outlets such as scalar fields- see Herdeiro et al contributions [13].

Now, we described the black hole solutions in our result which is as follows:

\section{Schwarzschild solution for Static Blackhole}

The Schwarzschild solution is the solution to Einstein Field Equation (EFE) that describes the gravitational field outside a spherical mass. In 1916 Karl Schwarchild found that the spherical rotation of the black hole is static means the electric charge and angular momentum of the mass and cosmological constant all are zero. It means that the uncharged static black hole is a black hole that has neither electric charge nor angular momentum. In the Schwarchild matric, the rotation of the black hole is stationary. A Schwarzschild black hole is described by the Schwarzschild metric(Carmine Cataldo, (2017).

Let us consider the spherical symmetry whose coordinates are $(t, r, \theta, \varphi)$ the usual static solution is described by the line element

$$
\begin{aligned}
& d s^{2}=-A(r) c^{2} d t^{2}+B(r) d r^{2}+r^{2} d \theta^{2}+r^{2} \sin ^{2} \theta d \varphi^{2} \\
& =A(r) c^{2} d t^{2}-B(r) d r^{2}-r^{2}\left(d \theta^{2}+\sin ^{2} \theta d \varphi^{2}\right)(2.1)
\end{aligned}
$$

Here A and B are asymptotically flat co-ordinate which is denoted by $r$ and $d \theta^{2}+\sin ^{2} \theta d \varphi^{2}$ is the static spherically symmetric metric.

From the eq. (2.1) the metric tensor is follows

$$
\begin{aligned}
g_{\mu v} & =\left[\begin{array}{cccc}
A(r) & 0 & 0 & 0 \\
0 & -B(r) & 0 & 0 \\
0 & 0 & -r^{2} & 0 \\
0 & 0 & 0 & -r^{2} \sin ^{2} \theta
\end{array}\right] \\
g^{\mu v} & =\left[\begin{array}{cccc}
\frac{1}{A(r)} & 0 & 0 & 0 \\
0 & -\frac{1}{B(r)} & 0 & 0 \\
0 & 0 & -\frac{1}{r^{2}} & 0 \\
0 & 0 & 0 & -\frac{1}{r^{2} \sin ^{2} \theta}
\end{array}\right]
\end{aligned}
$$

Let we infer the Christoffel symbol, we have 


$$
\Gamma_{\mu v}^{w}=\frac{1}{2} g^{w h}\left(g_{h \mu, v}+g_{h v, \mu}-g_{\mu v, h}\right)
$$

If the indices are oriented from 0 to 3, then 0 stands for $\mathrm{t}, 1$ stand for $\mathrm{r}, 2$ stands for $\theta$ and 3 stands for $\varphi$

Now, if we put $w=0$, then from equations (2.2),(2.3) and (2.4), we get

$$
\Gamma_{01}^{0}=\Gamma_{10}^{0}=\frac{1}{2 A} \frac{d A}{d r}
$$

If $w=0$ then all the other symbols are vanishing.

If we put $w=1$ then from equations (2.2), (2.3) and (2.4)

$$
\Gamma_{00}^{1}=\frac{1}{2 B} \frac{d A}{d r}, \quad \Gamma_{11}^{1}=\frac{1}{2 B} \frac{d B}{d r}, \quad \Gamma_{12}^{1}=\frac{-r}{B}, \quad \Gamma_{13}^{1}=\frac{-r}{B} \sin ^{2} \theta
$$

If $w=1$, then all the other symbols are vanishing.

If we put $w=2$, then from equations (2.2), (2.3) and (2.4), we get

$\Gamma_{12}^{2}=\frac{1}{r}=\Gamma_{21}^{2}, \quad \Gamma_{33}^{2}=-\sin \theta \cdot \cos \theta$

If $w=2$, then all the other symbols are vanishing.

If we put $w=3$, then from equations (2.2), (2.3) and (2.4), we get

$\Gamma_{13}^{3}=\frac{1}{r}=\Gamma_{31}^{3}, \Gamma_{23}^{3}=\frac{1}{\tan \theta}=\Gamma_{32}^{3}$

If $w=3$, then all the other symbols are vanishing.

Now, let us infer the components of the Ricci tensor

$R_{\mu v}=\frac{\partial \Gamma_{\mu w}^{w}}{\partial x^{v}}-\frac{\Gamma_{\mu v}^{w}}{\partial x^{w}}+\Gamma_{\mu w}^{h} \Gamma_{v h}^{w}-\Gamma_{\mu v}^{h} \Gamma_{w h}^{w}$

We get all the components are non-vanishing, now from the equations (2.2), (2.3) and (2.9)

If $\mu=0$ and $v=0$, we get

$R_{00}=\frac{-1}{2 B} \frac{d^{2} A}{d r^{2}}+\frac{1}{4 B} \frac{d A}{d r}\left(\frac{1}{A} \frac{d A}{d r}+\frac{1}{B} \frac{d B}{d r}\right)-\frac{-1}{r B} \frac{d A}{d r}$

If $\mu=1, v=1$, we get

$R_{11}=\frac{1}{2 A} \frac{d^{2} A}{d r^{2}}-\frac{1}{4 A} \frac{d A}{d r}\left(\frac{1}{A} \frac{d A}{d r}+\frac{1}{B} \frac{d B}{d r}\right)-\frac{1}{r B} \frac{d B}{d r}$

If $\mu=2, v=0$, we get

$R_{22}=\frac{1}{B}+\frac{r}{2 B}\left(\frac{1}{A} \frac{d A}{d r}-\frac{1}{B} \frac{d B}{d r}\right)-1$

If $\mu=3, v=3$, we get

$R_{33}=\sin ^{2} \theta\left[\frac{1}{B}+\frac{r}{2 B}\left(\frac{1}{A} \frac{d A}{d r}-\frac{1}{B} \frac{d B}{d r}\right)-1\right]=\sin ^{2} \theta R_{22}$

Further, now we can write the EFE as follows

$R_{\mu v}-\frac{1}{2} R g_{\mu v}=\frac{8 \pi \varepsilon}{c^{4}} T_{\mu v}$

Here $\mathrm{R}$ intimates the Ricci tensor and $T_{\mu v}$ intimates the stress-energy tensor and $g_{\mu v}$ intimates the metric tensor.

If we consider the mass which has neither matter nor energy, then Einstein tensor must vanish.

Consequently, we have 


$$
R_{\mu v}-\frac{1}{2} R g_{\mu v}=0
$$

From equation (2.15) the Einstein tensor and Ricci tensor both are trace reverse to each other, we have $R_{\mu v}=0$

From equations (2.10), (2.11) and (2.12), we get

$\frac{-1}{2 A B} \frac{d^{2} A}{d r^{2}}+\frac{1}{4 A B} \frac{d A}{d r}\left(\frac{1}{A} \frac{d A}{d r}+\frac{1}{B} \frac{d B}{d r}\right)-\frac{1}{r A B} \frac{d A}{d r}=0$

$\frac{1}{2 A B} \frac{d^{2} A}{d r^{2}}-\frac{1}{4 A B} \frac{d A}{d r}\left(\frac{1}{A} \frac{d A}{d r}+\frac{1}{B} \frac{d B}{d r}\right)+\frac{1}{r A B} \frac{d A}{d r}=0$

Now, if we dividing the equation (2.17) and (2.18), we get

$$
\begin{aligned}
& \frac{d B}{B}=\frac{-d A}{A} \\
& B=\frac{C_{1}}{A}
\end{aligned}
$$

$C_{1}$ is the constant, the solution will be asymptotically flat, if we use the Minkowski space for $r \rightarrow \infty$, then we interpolate the boundary conditions.

$$
\lim _{r \rightarrow \infty} A(r)=\lim _{r \rightarrow \infty} B(r)=1
$$

From (2.20) taking for (2.21), we get

$$
\begin{aligned}
& B=\frac{1}{A} \\
& g_{00} g_{11}=-1
\end{aligned}
$$

Put $B=\frac{1}{A}$ in equation (2.12) and from equation (2.12) and (2.16)

$$
\begin{aligned}
& A+\frac{r A}{2}\left[\frac{1}{A} \frac{d A}{d r}-A \frac{d}{d r}\left(\frac{1}{A}\right)\right]-1=0 \\
& A+r \frac{d A}{d r}-1=\frac{d}{d r}(r A)-1=0 \\
& A=1+\frac{C_{2}}{r}
\end{aligned}
$$

It is worth noting that, we could already infer the original Schwarchild solution [9], without defining any particular value of the constant $C_{2}$.

If we intimate with $\varphi$ the Newtonian potential, we can write

$$
A=g_{00} \approx 1+\frac{2 \varphi}{c^{2}}=1-\frac{2 M G_{N}}{r c^{2}},
$$

Where $G_{N}$ is the Newtonian constant of gravity, c is the velocity of light and $\mathrm{M}$ is the point mass at the origin

Now from (2.26) and (2.27), we have

$$
C_{2}=\frac{-2 M G_{N}}{c^{2}}
$$

and from (2.22) and (2.27), we get

$$
B=\frac{1}{1-\frac{2 M G}{c^{2}}}
$$

With the help of equations (2.27) and (2.29), we get a metric 


$$
d s^{2}=-\left(1-\frac{2 M G_{N}}{c^{2} r}\right) c^{2} d t^{2}+\frac{d r^{2}}{1-\frac{2 M G_{N}}{r c^{2}}}+r^{2}\left(d \theta^{2}+\sin ^{2} \theta d \varphi^{2}\right)
$$

Here, we show that the metric appearance to have a singularity of the Schwarzschild radius $r_{s}=\frac{2 M G_{N}}{c^{2}}=2 m$. The above equation can also be written as

$d s^{2}=-\left(1-\frac{2 m}{r}\right) c^{2} d t^{2}+\left(1-\frac{2 m}{r}\right)^{-1} d r^{2}+r^{2}\left(d \theta^{2}+\sin ^{2} \theta d \varphi^{2}\right)$

Here, $d s^{2}$ has a glaring singularity at $r=2 m$ and co-ordinate $t$ has range $-\infty<t<\infty$.

The above solution is the unique solution of vacuum EFE. Which is describes the vacuum space-time outside a spherically symmetry object of mass M. While this object could have a time-dependent mass distribution and the external space-time is necessarily static and its metric is given by equation (2.30), so the equation (2.30) is the Schwarchild static matric. Further, the Schwarzschild metric is static if the intellect that, $\nabla$ is a timelike Killing vector which is gradient. Here, $t$ is a time-independent component of metric $g_{\mu v}$. Also, in equation (2.30), there are no mixed terms involving both space and time. Hence there is no rotation inherent in space-time.

Now, we discuss here briefly the EFE have stationary and static solutions. If the time doesn't enter explicitly in the metric potentials, then the solution is called stationary. In such a case, the components of metric will be time-independent then the co-ordinate system will existi.e., if $t$ is the time like coordinate

$$
\frac{\partial g_{\mu v}}{\partial t}=0
$$

In this coordinate system, the Lie derivative $\mathcal{L}_{X} g_{\mu v}=0$ and we define the vector $X^{i}=\delta_{0}^{i}$ i.e., Lie derivative vanishes in all co-ordinate systems for the Killing vector $X^{i}$. On the other hand, if the spacetime assumes a time like Killing vector field, then it is possible to choose a coordinate system adapted to it such that $\mathcal{L}_{X} g_{\mu v}=0$. Then the metric is again stationery andspace-timeare called stationary if and only if it admits the existence of a time likeKilling vector fields.

We note that if space-time is stationary, it does mean that the components of metric cannot develop in time. It is just that the time doesn't enter clearly in the solution. However, the stronger requirement of staticity means that there is no time evolution of the system, which is time-symmetric about any origin of time. In such a way, one would expect that in the co-ordinate system conditioned to the time like Killing vector field, the metric would accept no cross-terms as well such a way under a time-reversal $t \rightarrow-t$, the sign of those elements of $d s^{2}$ containing the cross terms in $g_{\mu v} \rightarrow-g_{\mu v}$ will be reversed. However, the stativity assumption means that the $d s^{2}$ must remain invariant under the $t \rightarrow-t$ about any origin of time. This implies that the cross terms must vanish in the manifestation of $d s^{2}$. Hence a static solution of space-time is classified by the existence of a time like vector $t$ Killing vector field 
for the space-time, and the additional requirement that in the co-ordinate system conditioned to this vector field the components of metric are time-independent and no cross-terms appear in the line element $d s^{2}$. Hence asdiscussed above, the Schwarzschild metric (3.30) is the solution of the vacuum EFE with the assumption of spherical symmetry in space-time. It is glaring that the coordinate system $(t, r, \theta, \varphi)$ provides a frame in which the metric is time-independent. So, the equation (3.30) is the static or stationary solution.

\section{Kerr-Newman Solution for Rotating Black Hole}

As stated in the previous section, the final configuration resulting from a complete spherically symmetrical gravitational collapse is defined by the Schwarzschild geometry in the exterior of the collapse dust cloud configuration as solutions to the Einstein equations. Even if the collapse is nonspherical and the geometry outside is time-independent on would expect the geometry to settle to a stationary final state in the end. Further, Ali celestial bodies rotate, and the resulting solution will not be assumed to be precisely spherically symmetrical. The Kerr space-time integrates these features and models the external geometry around a spinning body as well as a black hole that rotates (Kerr, 1963). The metric is written in the Boyer-Lindquist (1967) co-ordinates $(t, r, \theta, \varphi)$ as [6]

$d s^{2}=\frac{\Delta-a^{2} \sin ^{2} \theta}{\rho^{2}} d t^{2}+\frac{2 \chi M a}{\rho^{2}} r \sin ^{2} \theta d \psi d t-\frac{\rho^{2}}{\Delta} d r^{2}-\rho^{2} d \theta^{2}-\frac{A \sin ^{2} \theta}{\rho^{2}} d \psi^{2}$

$$
\text { Where } \Delta \equiv r^{2}-2 \chi \mu r+a^{2}
$$

Here $\mathrm{m}$ is the gravitational mass parameter of the body and $\mathrm{J}$ represents the total angular momentum of space-time i.e., $a=\frac{J}{M}$ or $J=M a$, it is the per unit mass angular momentum of an object.

$$
\begin{aligned}
& \rho^{2}=r^{2}+a^{2} \cos ^{2} \theta \\
& A=\left(r^{2}+a^{2}\right)^{2}-a^{2} \Delta \sin ^{2} \theta
\end{aligned}
$$

So, one can see here that there are two parameters $M$ and an on which the black hole depends if we put $a \rightarrow 0$ then the black hole metric reduces to the Schwarzschild metric, as one can see as $r$ goes to infinity, we encounter the following metric tensor approximately

$d s^{2} \approx\left(1-\frac{2 \chi M}{r}\right) d t^{2}+\frac{2 \chi \mu a \sin ^{2} \theta}{r} d \psi d t-\left(1+\frac{2 \chi M}{r}\right) d r^{2}-r^{2} d \Omega^{2}$

Where $\mathrm{M}$ is a mass because it was out this term this just reduces to the same form as a Schwarchild does it reduce because this when $a=0$ this reduces to Schwarchild, so $\mathrm{M}$ is a mass and a is the presence of this term of equation (3.1) because it the presence of this metric although it invariant under the time translations $t \rightarrow t+a$ but it is not invariant under the time-reversal $\rightarrow-t$ exactly because of the presence of this term of equation (3.1) this term makes this metric stationery but not static(Eva Hackmann,2010). So, its components of the metric are time-independent but there are nondiagonal terms. This term is correspondence to the rotational terms. So, the space-time describes the rotation so $\tau=M a$ the physical meaning of $a$ is momentum attributed for angular momentum per unit mass. 
Now, let us see with this metric has a horizon or not, first of all, to do that explicitly to see Penrose diagram

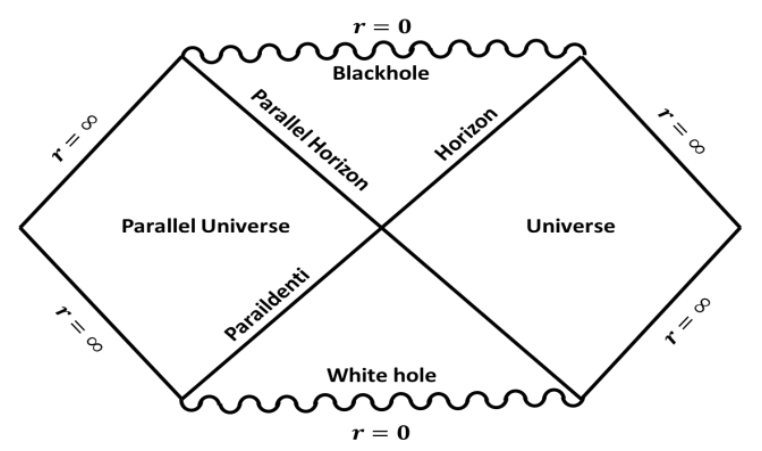

Fig 3.1 Newman-Penrose diagram

For $\frac{\rho^{2}}{\Delta^{2}} d r^{2}$ metric, the Schwarchild case for equation (3.2) if $\Delta=0$

$$
\begin{aligned}
& \Delta=r^{2}-2 \chi M r+a^{2} \\
& r^{2}-2 \chi M r+a^{2}=0
\end{aligned}
$$

Two solutions of this equation are

$$
r_{ \pm}=0 \text { and } \chi M \pm \sqrt{(\chi M)^{2}-a^{2}}=0
$$

As we can see $a \rightarrow 0, r_{+} \rightarrow r_{s}$ (Schwarzschild radius), here $r_{+}$is the radius of the horizon.

Now, on the way to see the Penrose diagram for this solution and another way to see that is considering normal vector for this surface(Pankaj S. Joshi, 1993).

$$
r=r_{+} \text {time like, }(\eta \mu)^{2}=0 .
$$

This is the horizon because of the escape of this surface so this is an invariant horizon.

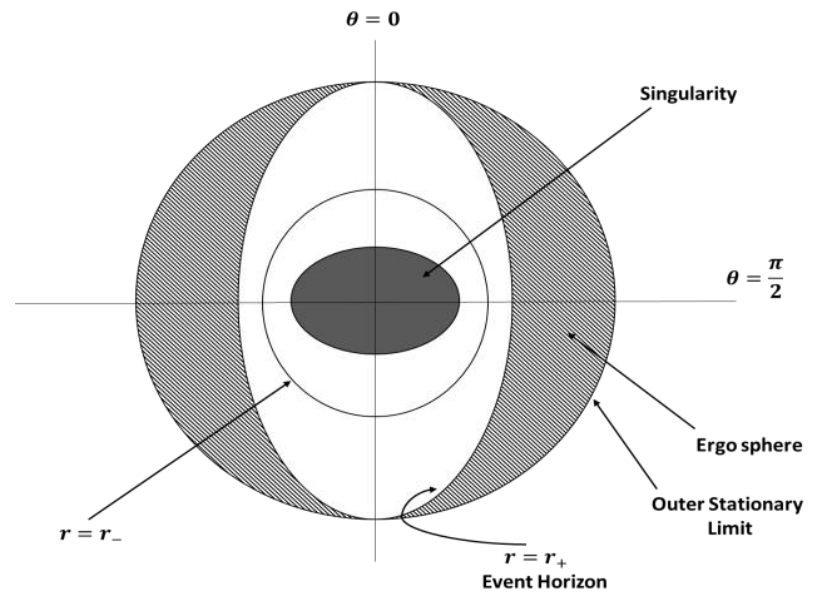

Fig 3.2 The Stationary unit surface and event horizon in Kerr solution with $0<a^{2}<\boldsymbol{m}^{2}$

If we vanish the term $\frac{\Delta-a^{2} \sin ^{2} \theta}{\rho^{2}} d t^{2}$ from the equation (3.1) then the solution of this term is

$$
r_{0}(\theta)=\sqrt{(\chi M)^{2}-a^{2} \cos ^{2} \theta}
$$




$$
\text { if } r_{0}(\theta) \geq r_{+}
$$

We counted the following situation now, the real solution for equation (3.7) $\chi M>a$. In a real situation during the collapse, one can't create such an object with $\chi M<a$

When $\chi M=a$ is called a critical situation. More generally its states that one during real, physical situation one can't create space-time which is a singularity that is not surrounded by the horizon.

Let us explained that on the Schwarchild Penrose diagram it can be seen. If we have anyone who has stayed very close to the black hole horizon it can stay fixed at the fixed radius and if some seeing inside the black hole creates radiation some craze motion like masses or charges any radiation will go to torque singularity it will not go outside of this surface. So, their Schwarchild horizon is the horizon of the black hole in the surface of infinite redshift.

Let us study it from a collapse if we have a collapse where the matter of the black hole falls down the creation of their horizon for approaches its horizon and during the collapse process is happening so many singularities exist but as Schwarchild solution it very closed to the horizon.

Suppose you have an Electromagnetism you have an ideal sphere which is homogeneous with distributed electric charge over it this sphere is breathing so it like goes outside and so we shrink and expand respecting spherical symmetry and homogeneity of the charge distribution independently of the radius of this sphere it always creates outside of its coulomb field so the electric field is not zero but a magnetic field is zero. So, it means that such a sphere even though the charges are moving with acceleration in the motion. This breathing sphere never creates electromagnetic radiation also it of field its known by those who are building antennas they know that spherically symmetric antenna cannot be created so that is related to the fact that to have electromagnetic radiation you have to have dipole moment changing in time while for the breathing sphere dipole momentum with respect to its centre is always zero and actually all multiple momentum with respect to its centre as zero so similar situation we have in gravity and even more defined in the sense that spherically symmetric mass when it breezes doesn't create gravitational radiation because outside of it in Newton gravity creates Newton fields in Einstein gravity it creates due to Birkoff theorem and create Schwarchild field so these are very interesting situations so during collapse process everything that can be radiated it ready that remains in the object which is characterized only by three charges by mass, angular momentum and electric field by electric charge(Pankaj S. Joshi,1993).. So, these are the only charge which cannot create electromagnetic or gravitational radiation all the rest of the multiple momenta can create radiation and they are radiated so this fact in the bases of the no-hair theorem in the absence of the electric field when we don't have this, we have the solution which is characterized only by two parameters by mass and angular momentum as a result of the collapse process that is a statement of the no-hair theorem. 


\section{Reissner-Nordstrom Solution for Static and Charge Blackhole}

The final condition of a complete gravitational collapse, either spherically symmetrical or nonspherically symmetrical, is considered to be a space-time vacuum containing the rotation and probably also the electromagnetic fields associated with the body. The charge associated with an astrophysical object may be quickly neutralized by the surrounding plasma. In any case, however, it would be of interest to obtain Ali solutions to Einstein-Maxwell equations which could explain with charge stationery collapsed configurations. The charged generalization of Schwarchild geometry is called the Reissner-Nordstrom metric, describing the space-time outside of a spherically symmetrical electrically charged body. This is a static and asymptotically charged solution of the EinsteinMaxwell equations $G_{\mu v}=8 \pi T_{\mu v}$. Here $T_{\mu v}$ is the trace free; Maxwell energy-momentum tensor $F_{\mu v}$ satisfies the Maxwell equation in the source-free region [3].

For the metric coordinates $(t, r, \theta, \varphi)$ is written as follows

$$
d s^{2}=-\left(1-\frac{2 M}{r}+\frac{q^{2}}{r^{2}}\right) d t^{2}+\frac{d r^{2}}{1-\frac{2 M}{r}+\frac{q^{2}}{r^{2}}}+r^{2}\left(d \theta^{2}+\sin ^{2} \theta d \varphi^{2}\right)
$$

The above metric is the Reissner-Nordstrom metric. This is a time-independent and spherically symmetric metric.

Here $\mathrm{M}$ is the gravitational mass and $\mathrm{q}$ is the electric charge of the body. The $T_{\mu v}$ energy stress tensor doesn't vanish anymore. The exterior represented by this solution but corresponds to the electromagnetic field in space-time resulting from the charge of the body. Here we can again establish an analogy with Birkoff's theorem, namely, this is the spherically symmetrical solution of the Einstein-Maxwell equations. It's always unchanged.

If a local orthonormal bases generated

$$
\begin{aligned}
& e_{\hat{t}}=\left(1-\frac{2 M}{r}+\frac{q^{2}}{r^{2}}\right)^{1 / 2} e_{t} \\
& e_{\hat{r}}=\left(1-\frac{2 M}{r}+\frac{q^{2}}{r^{2}}\right)^{1 / 2} e_{r} \\
& e_{\hat{\theta}}=\frac{1}{r} e_{\theta} \\
& e_{\hat{\varphi}}=\frac{1}{r \sin \theta} e_{\varphi}
\end{aligned}
$$

An observer that is stationary to the co-ordinate system then sees the outward-pointed observant (4 velocity $u=e_{\hat{t}}$ ) magnitude electric field $E_{\hat{r}}=\frac{q}{r^{2}}$. The strength of the electromagnetic field is

$$
\begin{aligned}
& F_{t r}=\frac{-q}{r^{2}}, F_{r t}=\frac{q}{r^{2}}, \text { all other components are zero. } \\
& A_{t}=\frac{-q}{r}, \text { other components are zero }
\end{aligned}
$$

The efficiency of the Reissner- Nordstrom solution is based on the relationship between electric charges $\mathrm{Q}$ and the mass $\mathrm{M}$. define the function 
$f(r)=1-\frac{2 M}{r}+\frac{q^{2}}{r^{2}}$, we can see that:

i. If $|q|<m$, then $f(r)$ has two zeroes at $r_{ \pm}=m \pm \sqrt{m^{2}-q^{2}}$. The exterior feels qualitatively close to the outside Schwarchild space-time. In particular, if we take $q \rightarrow 0$, then the uniqueness of the outermost co-ordinates occurs at $r_{+}=2 \mathrm{~m}$

ii. If $|q| \geq m$, then all is well done at positive $r[f(r)>0]$. The central singularity at $r=0$ (which is, like, in Schwarchild, there is a physical singularity) visible to the outside world as a naked singularity.

\section{Conclusion}

Here is the brief outline of the outcomes of the proposed research work:

1. In the introductory section, we give Newton theory of gravity, Einstein's special and general theory of relativity, and some fundamental aspects and discovery of wellknown black hole solutions.

2. In the second section, we use the Schwarzschild metric, some tensors like Ricci tensor and Einstein tensor,and study Schwarchild solution for the static black hole. With the help of this, we describe that the Einstein field equation has stationary and static solutions and components of metric will be time-independent.

3. In the third section, we describe the KerrNewman solution for rotating black hole and show that there are two parameters $\mathrm{M}$ and $a$ on which the black hole depends if we put $a \rightarrow 0$ then the black hole metric reduces to the Schwarchild metric. In Schwarchild Penrose diagram we see anyone who has stayed very close to the black hole horizon can stay fix at the fixed radius and if some seeing inside the black hole creates radiation some craze motion like masses or charges by radiation will go to torque singularity.

4. The Fourth section emphasis ReissnerNordstrom solution for static and charge black hole and define the relationship between electric charges $\mathrm{Q}$ and the mass $\mathrm{M}$.

\section{References}

Adamo Tim and Newmann ET (2016). The Kerr-Newman metric: A Review. Scholarpedia 9 (10): 31791.

AmselAaron J, GT Horowitz et al(2010). Uniqueness of Extremal Kerr and Kerr-Newman Black holes.

Physical Review D- Part. Fields, Gravit. Cosmol.81, Vol-2, pp. 024-033

Blau Matthias (2014). Lecture notes on general relativity. Albert Einstein centre for fundamental physics, Institute of Theoretical Physics. Bern University, $\quad \mathrm{CH}-3012$ Bern, Switzerland.

Cataldo C (2017). On the Schwarchild Solution: A Review. Int. J. Adv. Eng. Res. Sci. Vol-4, issue- 9, pp. 48-53

Chandrasekhar S (1998). The Mathematical Theory of Black holes. Clarendon 
Press. Oxford Univ. press. New

York

Hackmann Eva (2010). Geodesic equations in

Blackhole space-times with

Cosmological constant, Univ. of

Bremen.

Heinicke Christian and Hehl FW (2015).

Schwarzschild and Kerr Solutions of

Einstein's Field Equation- an

introduction. Int. J. Mod. Phys. D 24,

1530006 pp. 78

HirataCM (2012). Reissner-Nordstrom Black

holes. Caltech M/C 350-17, Pasadena

CA 91125, USA.

Joshi PS (1996). Global Aspects in gravitation

and Cosmology. Clarendon Press.

Oxford.

Vishwakarma RG (2016). Einstein and Beyond: A Critical Perspective on General Relativity. Universe, 2, $1-23$.

VojinovieMarko (2010). Schwarchild solution in General Relativity.

Walters S (2016). How Einstein Got his Field Equations. In commemoration of General Relativity's centennial,

W John. Norbury (1997). General Relativity and Cosmology, Univ. of WisconsinMilwaukee. 